\title{
A Walking Beacon-Assisted Localization in Wireless Sensor Networks
}

\author{
Bin $\mathrm{Xiao}^{1}$ \\ ${ }^{1}$ Department of Computing \\ Hong Kong Polytechnic University, HK \\ csbxiao@comp.polyu.edu.hk
}

\author{
Hekang Chen ${ }^{1,2}$, Shuigeng Zhou ${ }^{2}$ \\ ${ }^{2}$ Dept. of Computer Science and Engineering \\ Fudan University, Shanghai, China \\ \{hkchen, sgzhou\}@fudan.edu.cn
}

\begin{abstract}
The localization of the sensor node is a fundamental problem in sensor networks and can be implemented using powerful and expensive beacons. Beacons, the fewer the better, can acquire their position knowledge either from a GPS device or by virtue of being manually placed. In this paper, we propose two distributed methods to localization of sensor nodes using a single moving beacon where sensor nodes compute their position estimate based on the range-free technique. The first method uses the arrival and departure information of a walking beacon and the second method exploits the variance of the Received Signal Strength (RSS) from the beacon. We provide the upper bound of the estimation error for these methods in an ideal environment. Critical to the location accuracy of sensor nodes are two more parameters, the radio transmission range of the beacon, and how often the beacon broadcasts its position. Simulation results show the location estimate error of sensor nodes applying the proposed two methods. The results are consistent to the theoretical analysis and the average estimate errors could be within one meter.
\end{abstract}

\section{INTRODUCTION}

Wireless sensor networks (WSN) are a current focus of research interest because of their broad applicability in areas such as environmental observation, military monitoring, building monitoring and disaster relief. The performance of WSNs is crucially influenced by how accurately sensor nodes within the network are localized. Sensor localization information is used in the self-organization and configuration of networks, in deciding where events take place, tracking moving targets [1], [2], assisting traffic routing [3], [4] and providing the network geographic coverage [5].

Central to such location aware operations are the techniques used to identify the position of each sensor node. Constraints of cost and power consumption make it infeasible to equip each node in a network with a Global Positioning System (GPS) [6]. It is possible, however, to equip a small number of sensors. Such sensors nodes, in this paper referred to as beacons - may be static or mobile and may be used to identify the position of other nodes in a sensor network. This is done with either the range-based or the range-free technique. The range-based technique [7], [8], [9], [10] can detect the position of a node through the Received Signal Strength (RSS) measurement. RSS is a fundamental property to reflect the distance between two sensor nodes in the wireless communication environment. The equipped RSSI (Received Signal Strength Indicator) in many sensor platforms, such as mica2 and micaz, makes the direct distance measurement possible. However, the signal capture can be severely affected by the environmental interference, greatly degrading detection accuracy. In the range-free technique [11], [12], [13], each sensor node uses signals from a few beacons to calculate its approximate location. To directly receive signals, a node must fall inside the overlapping transmission area of several beacons. Nonetheless, the information that a node is outside of the transmission area of a beacon can also be used to set a tighter bound on the location of a node.

The moving beacon can be effectively utilized to locate the position of sensor nodes in WSNs [14], [15], [16], [17]. If the beacon moves and periodically broadcasts its position to nodes in its vicinity then a single beacon will suffice. Such a position-aware beacon can acquire its geographical position through GPS or the position can be known because it moves along a predefined route. Using a moving beacon that knows its position is broadly equivalent to using many stationary beacons each broadcasting once. A sensor node can compute an area to confine its location if it receives the coordinate messages from the beacon in a couple of times. Once a sensor node has approximately determined its position it can help to localize its neighbor nodes or even distant nodes. Ultimately, the location estimate of each sensor node is determined by the moving beacon. Thus, the degree of accuracy with which a sensor node is located is determined by the knowledge of the route that the beacon travels and how often it emits a signal.

In this paper, we propose two distributed methods to localization of sensor nodes using a moving beacon. The first method uses the arrival and departure information of a walking beacon and the second method exploits the variance of the RSS from the beacon. A sensor node locally computes its position estimate based on the range-free technique. Note that the beacon can have a prior knowledge of the deployment area of sensor nodes and thus can move in straight lines to cover the whole area. We provide the upper bound of the estimation error for two methods in an ideal environment. Critical to the location accuracy of sensor nodes are two parameters, the radio transmission range of the beacon, and how often the beacon broadcasts its position.

These methods do not require specially designed hardware equipments (e.g., angle detection, time synchronization) in sensor nodes in the localization context. They are robust to be applied practically in the real life and are scalable to 
locate each node given that the beacon can move to each corner of the sensor network deployment area, no matter that the sensor network topology is isotropic or anisotropic [18]. Simulation results show the location estimate error of sensor nodes applying the proposed two methods. The results are consistent to the theoretical analysis and the average estimate errors could be within one meter.

This paper is organized as follows. In Section II, we describe the related work in the area of sensor node localization. Section III and IV present the distributed localization techniques using the arrival and departure, RSS information of a moving beacon respectively. Section V demonstrates the simulation results of these methods in terms of RMSE (Root Mean Square Error) and Maximum estimate errors. Finally, we offer our conclusion in Section VI.

\section{RELATED WORK}

Most localization methods are static. That is to say, both the sensor network and the beacons are static. These static localization methods are either range-based or range-free. Range-based localization first uses RSS, AOA, TOA or TDOA to measure the distance or angle between an unknown node and a beacon, and then employs trilateration, triangulation or maximum likelihood to estimate the position of the unknown node. These methods have two significant drawbacks: 1) they require costly additional hardware support, 2) fading and noise can cause distance measurement errors [19]. Range-free localization methods are meant to overcome these drawbacks as they do not require the hardware support for measuring distances or angles. Instead, they exploit the communication and sensing ranges.

RSS roughly indicates the transmission range between two sensor nodes and is an important parameter to be utilized in both the range-based and range-free localization methods. How precisely RSS can be used to indicate the real transmission range is defined by the RSS model. The model [20] calculating RSS reflects the path loss using parameters, such as a referenced distance, path loss exponent and a random variation. Many range-based localization methods [8], [9], [10] directly use it to estimate the distance between two sensor nodes. However, the radio transmission is always unstable caused by signal fading and background noise, which could create heavy variance of RSS. As a result, the distance measurement by RSS will have significant error, which ultimately leads to the position estimation unaccuracy. Rangefree methods [11], [12], [13] are also using RSS to localize sensor nodes in WSNs. RSSs in these methods are not for estimating absolute distances. Instead, these methods compare the value of RSS with each other to capture the relative distance relationship among nodes. Tian He et al. [12], [13] propose an area-based algorithm named APIT that, if nodes are in the triangular regions constructed by beacons, refines the area where nodes might be located. In order to judge whether a node falls in the a triangular region, it does RSS comparisons between itself and its neighbors. In [11], each unknown node sorts the beacons in descending order of RSS measurement. The position that fits the sorted order roughly represents the location of the node. Satisfactory performance of these methods, however, requires a high ratio of beacons to nodes and a dense node connectivity.

Mobile localization [14], [15], [16], [17], [21] using moving beacons avoids the problems of static localization methods and is practical. Galstyan et al. [21] propose a distributed online algorithm using a moving beacon to localize static sensor networks. This is extended to a general model using an unknown target, in which radio communication and sensing constraints minimize the area where a node might be located. In [17], the authors present a localization scheme (REKF) with the assist of some mobile robots in a Delay-Tolerant Sensor Network (DTN). The similar work to ours can be found in [16]. It presents the real usage of a moving beacon containing a GPS system to broadcast its positions. Each node computes its location estimate according to either the broadcasting positions of the moving beacon or the positions of its neighbors. Neither of these methods, however, present the theoretical analysis about the maximum estimation error or improvement of robustness in the real environment. Our proposed methods differ significantly from previous work because they are rangefree methods employing a single powerful moving beacon, are robust and scalable, and can be applied to a sensor network of any connectivity.

\section{Distributed Localization Using ARrival AND DEPARTURE OF A MOVING BEACON}

In this section, we present a range-free distributed localization method ADO that uses a mobile beacon to estimate the positions of sensor nodes in the ideal environment. Note that if a node can receive a signal from the beacon, their distance is within radio transmission range $r$ of the beacon. We first study how a node uses the broadcasting signal message from the beacon to confine its estimation area when the beacon is moving in a straight line. Then we will show the upper bound of the estimation error to the real position of a node.

\section{A. Arrival and Departure of the Beacon}

Assume that a beacon moves in a straight line in the deployment area of a sensor network and at a certain distance interval called the broadcasting interval $s$ broadcasts a message containing its current position. The position of the beacon denotes the physical point at which it broadcasts a message. We also assume that a node can receive messages from the beacon only if it is inside the transmission range of the beacon. We define two states for every node: in and out. We also define two dynamic transitions from these two states: arrival and departure.

- out: The node is out of the broadcasting range of the beacon.

- in: The node is within the broadcasting range of the beacon.

- arrival: A node receives the current scheduled signal of beacon, but did not receive the scheduled signal from the beacon's previous position. The status of the node is set 
from out to in. The beacon is now at the arrival position. Its previous position is called the prearrival position.

- departure: A node received the preceding scheduled signal from the beacon, but does not receive the scheduled signal from the current position of the beacon. The status of the node is set from in to out. The previous position of the beacon is called the departure position and the current position of the beacon is called the post-departure position.

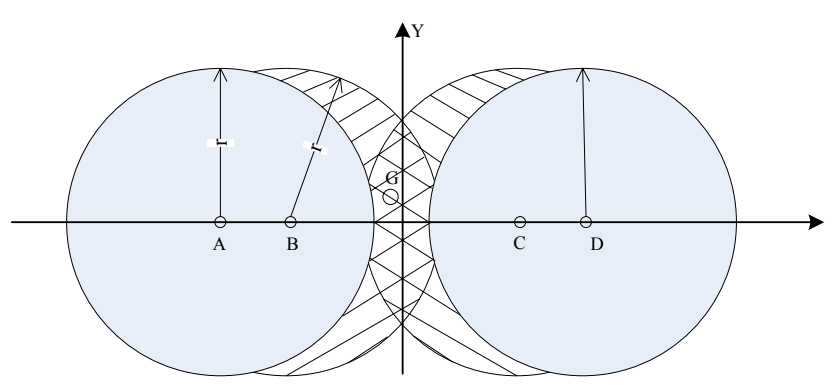

Fig. 1. Localization using arrival and leaving information of the beacon.

These two transitions are useful for estimating the position of a sensor node. The fact that transitions always occur in a pair limit the possible position of the node into a small area as shown in Figure 1. Suppose that the beacon is moving from the left to right along the $x$ axis. Initially, the state of node $G$ is set to out. When the beacon arrives at position $B$, node $G$ hears the beacon for the first time and its state is set to $i n$. Node $G$ can calculate the prearrival position $A$ according to the arrival position $B$. Node $G$ must fall inside the area in the circle centered at $B$ with radius $r$ and out of the circle centered at $A$, forming an arrival constraint area as in the left-hand crescent shape in Figure 1. When the beacon moves forward, it will walk out of the range of node $G$. Let $C$ be the departure position and $D$ be the post-departure position. The state of node $G$ is set to out and it must fall inside the area in the circle centered at $C$ with radius $r$ and out of the circle centered at $D$, forming a departure constraint area as the right-hand crescent shape in Figure 1. The arrival constraint area and departure constraint area will create an overlap called the Arrival and Departure Overlap (ADO). Thus, node $G$ must fall in $A D O$. The middle broadcasting positions between the arrival and departure positions do not provide valuable information because they cannot help to narrow down the area of $A D O$. To estimate its position, a node should obtain these four critical positions of the moving beacon: prearrival position, arrival position, departure position and post-departure position to compute its $A D O$.

We next provide the error upper bound of the position estimate of a node $G$ when transmission range is $r$ and broadcasting interval is $s$. Let the coordinates of node $G$ be $\left(x_{G}, y_{G}\right)$. Without loss of generality, we assume that the beacon starts moving along the $x$ axis from the coordinates $(0,0)$ and the node $G$ receives the first signal from the beacon when the beacon is at $(s, 0)$ and the last signal when the beacon is at $(k s, 0)$ where $k$ is the number of signals that the node hears between the arrival position and the departure position. The arrival constraint area of $G$ can be presented as:

$$
\begin{gathered}
x^{2}+y^{2}>r^{2} \\
(x-s)^{2}+y^{2} \leq r^{2}
\end{gathered}
$$

and the departure constraint area of $G$ can be presented as:

$$
\begin{gathered}
(x-k \cdot s)^{2}+y^{2} \leq r^{2} \\
(x-(k+1) \cdot s)^{2}+y^{2}>r^{2}
\end{gathered}
$$

where $1 \leq k \leq\lfloor 2 r / s\rfloor+1$, guaranteeing that the arrival constraint area and the departure constraint area overlap. It is obvious that the $A D O$ is symmetrical along the $x$ axis. The half $A D O(H A D O)$ above $x$ axis is called upper HADO while the other half $A D O$ is called lower $H A D O$. Assume that we can determine whether a detected node is above or below the line of movement of the beacon (we show the way in Section III-B). Without losing generality, we analyze the upper HADO to show the location estimate. Let the position estimate of node $G$ be at the point $O\left(x_{O}, y_{O}\right)$. As a result, the maximum horizontal error $M A X\left\{\left|x_{O}-x_{G}\right|\right\}$ and maximum vertical error $\operatorname{MAX}\left\{\left|y_{O}-y_{G}\right|\right\}$ depict the upper bounds of estimation error in the $x$ and $y$ coordinates respectively.

According to Formula (1) - (4), we calculate that the maximum horizontal error using node $O$ as the position estimate of node $G$ is

$$
\begin{cases}s / 2 & \text { if } s \leq k s \leq 2 r \\ \frac{2 r-(k-1) s}{2} & \text { if } 2 r<k s \leq 2 r+s\end{cases}
$$

and the maximum vertical error is

$$
\begin{cases}f(k-1)-f(k+1) & \text { if } s \leq k s \leq 2 r-s \\ f(k-1) & \text { if } 2 r-s<k s \leq 2 r+s\end{cases}
$$

where $f(k)=\frac{\sqrt{r^{2}-\left(\frac{k}{2} s\right)^{2}}}{2}$ and $k$ is the total number of signals that the node hears from the arrival position until the departure position. From Formula 5, it is obvious that the upper bound of the horizontal coordinate estimation error of node $G$ is equal to $s / 2$. From Formula 6 we know that $f(k-1)-f(k+1)$ is monotonously increasing whereas $f(k-1)$ is monotonously decreasing. Therefore, the upper bound of the vertical coordinate estimation error of node $G$ equals to $\frac{\sqrt{2 r s-s^{2}}}{2}$ when $k=\frac{2 r}{s}-1$ (in the real movement of a beacon, $k$ should be an integer and this upper bound still holds). The upper bound of the position estimation error of node $G$ decreases when $s$ decreases, which implies that to obtain an accurate localization of sensor nodes, the moving beacon should shorten its message broadcasting interval.

\section{B. Movement Pattern of Beacon}

In this subsection, we describe the movement pattern of a beacon that can cover and localize each sensor node accurately. The movement pattern is shown in Figure 2 in which the distance between two adjacent lines of movement is $r$.

In this pattern, a node can detect its position once or twice. The scenario in which a node can detect its position only once 


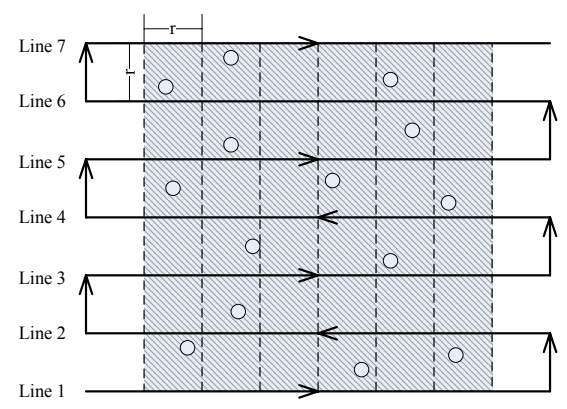

Fig. 2. The traverse route of the beacon for the ADO method.

happens when the node stays in the uncovered space that is not covered by two consecutive broadcasting of the beacon as shown in Figure 3(a). The node does not receive any signal from the above or below the lines and obtains one $A D O$ from the line across this uncovered space. The position of the $A D O$ can help to identify the uncovered space that the node resides in. Note that the uncovered space is symmetrical along the line of movement and we don't know whether the node is above or below the line. Because the uncovered space is relatively small, this space itself is a good position estimate of the node and it is not necessary to distinguish whether it is above or below. The $x$ coordinate of the center point of the $A D O$ is $\frac{k+1}{2} s$. We have two situations of the intersection of possible uncovered spaces with a verified $A D O$. The first situation is shown in Figure 3(b) where the $A D O$ intersects with only one uncovered space when $k+1$ is an odd number. We choose the center node $O$ in the uncovered space as the estimate position. The second situation is shown in Figure 3(c) where the $A D O$ intersects with two uncovered spaces when $k+1$ is an even number. To minimize the estimation error, we choose the connection point $O$ of two uncovered spaces as the estimate position. In both situations, the coordinates of node $O$ are $\left(\frac{k+1}{2} s, y_{\text {line }}\right)$ where $y_{\text {line }}$ is the $y$ coordinate of the line of movement.

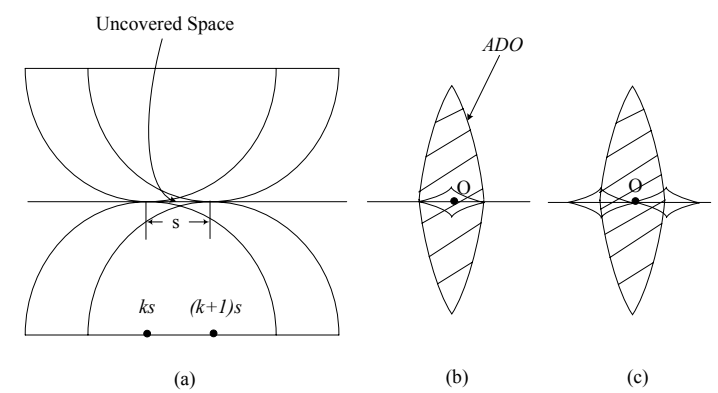

Fig. 3. Uncovered space when the beacon moves along a straight line.

The scenario in which a node detects its position twice happens for most occasions if the node stays outside of the small uncovered area. Each detection creates an estimate area for a node. This estimate area can be easily distinguished as the upper or lower HADO. A node hearing a signal from the $i$ th line is below the line when it has already received a signal from the $(i-1)$ th line. Otherwise, the node is above the line. Thus, a node in this scenario can be delimited into a more accurate area that is the overlap of two HADOs. These two HADOs provide a complementary estimate of a node to narrow down the possible area. In order to simplify the position estimate for nodes who obtain two $H A D O$ s, we first calculate the central point of the each $H A D O$ separately and then set their midpoint as the estimate position.

\section{Distributed Localization Using RSS OF A MOVING BEACON}

In this section, we present the RSS localization method when the beacon moves in a straight line in the ideal situation. RSSI (Received Signal Strength Indicator) is always available in many sensor platforms such as mica2 and micaz. The most widely used assumption in the ideal situation is that the shorter distance a sensor node resides from the beacon, the larger RSSI will be obtained. We do not use RSSI to estimate distance from beacon, instead, we utilize the variance of RSSI to estimate the node position. Thus, our RSS localization method also belongs to range-free technique.

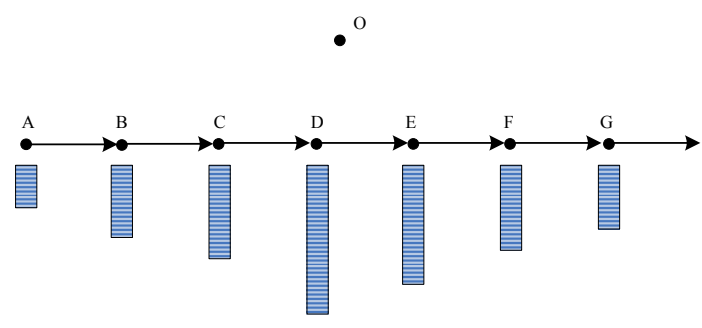

(a) The variance of RSS.

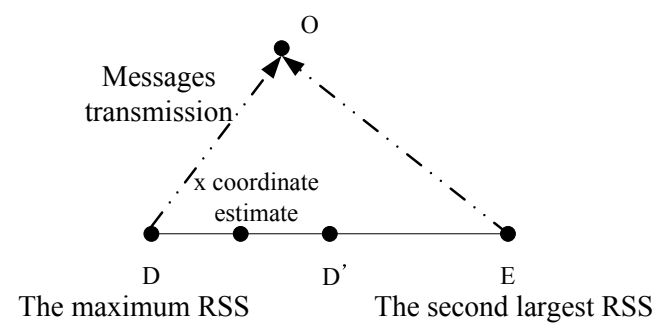

(b) The $x$ coordinate estimate of node $O$.

Fig. 4. Localization using RSS.

\section{A. Location Estimate}

The location estimate of a sensor node $O$ is derived from the variance of received RSS from the moving beacon. At first, node $O$ does not receive any signal from a distant beacon. When the beacon moves closer, the received RSS becomes stronger. After the beacon passes the point that is the nearest position to node $O$, RSS will decrease accordingly. Thus, node $O$ will get a RSS series. This series is shown in Figure 4(a) where the height of columns indicates the size of RSS. Figure 4(b) shows how to compute the position estimate of node $O$. Suppose that the beacon is moving along the $x$ axis with broadcasting interval to be $s$. The maximum signal strength received at node $O$ happens when the beacon is at point $D$ and the second maximum signal strength is at point $E$. Thus, the $x$ coordinate of node $O$ should be within $D$ and $E$. Furthermore, 
given that $D^{\prime}$ is the midpoint between $D$ and $E$, node $O$ can conclude that its $x$ axis coordinate is between point $D$ and $D^{\prime}$. For simplicity, we set the midpoint between $D$ and $D^{\prime}$ as the $x$ axis estimate of node $O$. Obviously, the maximum estimate error of the $x$ coordinate of node $O$ is $s / 4$ as the $x$ coordinate of node $O$ must reside in the interval $\left[D, D^{\prime}\right]$.

\section{B. Movement Pattern}

Figure 5 shows the RSS movement pattern for the RSS localization method. When the beacon is moving along horizontal lines, unknown nodes can only determine their $x$ coordinates. Thus, the beacon needs to move along vertical lines for nodes to estimate their $y$ coordinates. Given the RSS movement pattern as shown in Figure 5, we know that the maximum $y$ coordinate estimate error is the same as the $x$ coordinate, i.e., $s / 4$. This estimate error upper bound is smaller than that of the ADO method and only relevant to the broadcasting interval (the value of $s$ ). Although the RSS movement pattern is distinct from that of the ADO movement pattern, the total traveling distance of the beacon in both movement patterns are comparative. The disadvantage of the RSS method is that a small portion of nodes may not be localized if they reside in the uncovered space as denoted in Figure 3. To overcome this disadvantage, we can slightly shorten the distance between two moving straight lines of the beacon (smaller than $2 r$ ) to ensure that each sensor node can receive signals from the beacon.

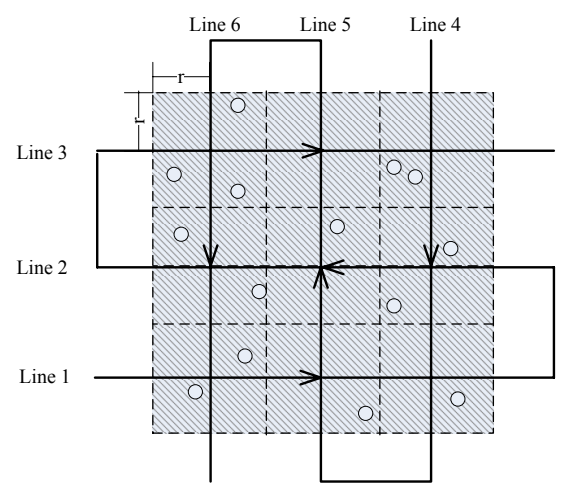

Fig. 5. The traverse route of the beacon for the RSS method.

\section{Performance Evaluation}

In this section, we evaluate the performance of the proposed two localization methods: ADO and RSS. The simulation is implemented as 50 nodes randomly distributed in a $16 \mathrm{~m} \times 16 \mathrm{~m}$ area and a moving beacon broadcasting its geographical position information with an adjustable transmission range $r$. The broadcasting interval of the beacon is $s$. The traverse routes of the beacon are given in Figure 2 and 5 for ADO and RSS respectively. We totally conduct three sets of simulations. The first and second sets investigate the performance with various broadcasting intervals and transmission ranges of the moving beacon in terms of the RMSE (Root Mean Square Error) and the maximum estimation errors. The third one studies the percentage of localized nodes out of all nodes in RSS.

Performance under various broadcasting intervals. In the first set of simulations, we study the position estimate errors, i.e., RMSE and the maximum error, of sensor nodes when the broadcasting interval of the beacon increases from $0.25 \mathrm{~m}$ to $1.5 \mathrm{~m}$ and its transmission range is $r=6 \mathrm{~m}$. Figure 6 shows that both localization methods (ADO and RSS) can yield accurate estimates where the real location of each sensor node can be confined within a distance (smaller than $1.6 \mathrm{~m})$ to the calculated location. The average estimate error is within $0.8 \mathrm{~m}$. The figure also illustrates that the RSS localization method can achieve smaller RMSE and maximum error than the ADO localization method does. The estimate errors increase as the broadcasting intervals of the beacon increase. The maximum errors of both methods are consistent to previously theoretical analysis where the coordinate upper bounds of estimate errors for a node is $\left(\frac{s}{2}, \frac{\sqrt{2 r s-s^{2}}}{2}\right)$ in the ADO method whereas $\left(\frac{s}{4}, \frac{s}{4}\right)$ in the RSS method.

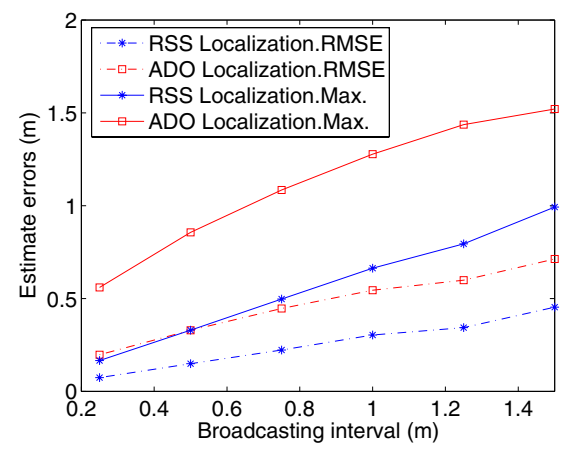

Fig. 6. Location estimate errors under various broadcasting intervals.

Performance under various transmission ranges. In the second set of simulations, we show the impact of the beacon's transmission range on the position estimation accuracy. We set the broadcasting interval $s$ to be $0.5 \mathrm{~m}$ and vary the transmission range from $1 \mathrm{~m}$ to $6 \mathrm{~m}$. Figure 7 shows that ADO can achieve satisfactory performance where the RMSE and maximum error are smaller than $0.4 \mathrm{~m}$ and $1 \mathrm{~m}$ respectively. When the transmission range goes larger, both the RMSE and maximum errors of ADO rise. The RSS method, however, outperforms ADO with smaller RMSE and maximum errors. Furthermore, the curves of RSS are almost flat, which indicates that RSS is insensitive to the variance of the transmission range.

Percentage of localized nodes in RSS. In the last simulation, we study the percentage of localized nodes in RSS under various broadcasting intervals and transmission ranges. Although RSS cannot guarantee that all nodes will be localized due to the uncovered space as described in Section IV-B, Figure 8 shows that the percentage of localized nodes 


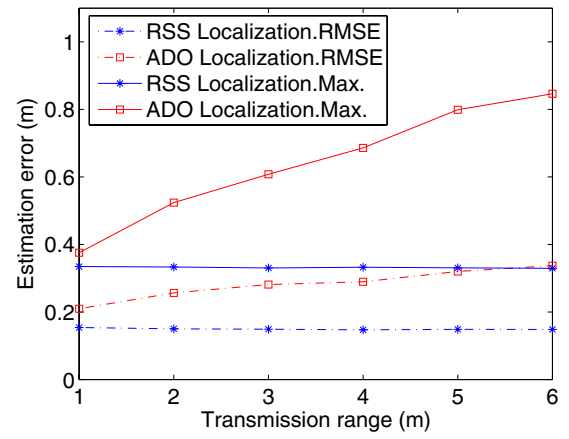

Fig. 7. Location estimate errors under various transmission ranges.

in RSS still reaches more than $98 \%$. When the broadcasting interval increases, the percentage drops as shown in Figure 8(a). This is because the larger broadcasting interval leads to bigger uncovered space. On the other hand, when we increase the transmission range, Figure 8 (b) shows that the percentage rises. The reason is that the increment of the transmission range reduces the number of uncovered spaces and thus reduces the number of non-localized nodes.

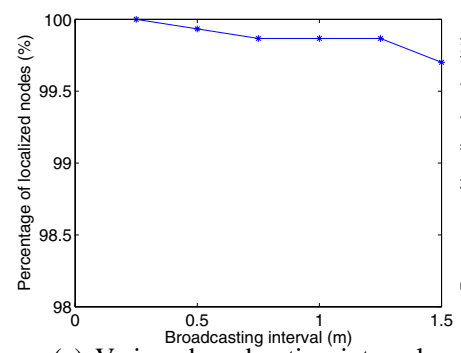

(a) Various broadcasting intervals

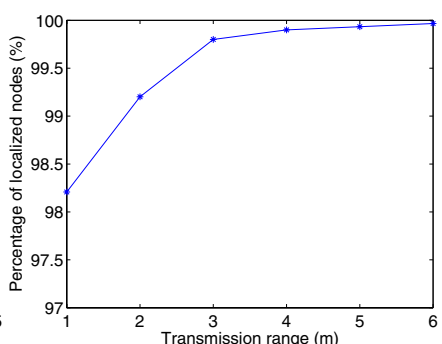

(b) Various transmission ranges
Fig. 8. Percentage of localized nodes in RSS.

\section{CONCLUSION}

In this paper, we present two distributed range-free localization methods (ADO and RSS) that use only one moving beacon within a sensor network. The basic idea of the ADO method is to narrow down the possible location of a node by using arrival and departure constraint areas derived from the moving beacon. The RSS method seeks the location of a sensor from a sequence of RSS broadcasted from the moving beacon. Both methods employ the range-free techniques in the sense that they do not rely on the direct distance measurement from RSS but on a sequence of constraints. We provide the upper bound of the estimation error for two methods in an ideal environment. Critical to the location accuracy of sensor nodes are two parameters, the radio transmission range of the beacon, and how often the beacon broadcasts its position. Another factor, transmission range, also affects the performance of the ADO method. The RSS method, however, is not sensitive to its varying. We conduct several simulations to evaluate these two methods. The simulations show that the results of both methods are consistent to theoretical analysis. Under various simulation scenarios, both methods perform well, with the average estimate error smaller than one meter. The RSS method outperforms the ADO method which can reduce the estimate error by $50 \%$. However, the disadvantage is that the RSS method cannot guarantee all nodes to be localized when the traverse route gap of the beacon is bigger or equal to $2 r$. In such condition, the localized nodes is still satisfying, with a percentage over $98 \%$.

\section{ACKNOWLEDGMENT}

This work is partially supported by HK RGC CERG BQ827, 2007 CB307100 China National 973 Program Grant, NSFC under grant no. 90612007, and by the Shuguang Scholar Program of Shanghai Education Development Foundation.

\section{REFERENCES}

[1] W. Zhang and G. Cao, "Dctc: Dynamic convoy tree-based collaboration for target tracking in sensor networks," IEEE Transactions on Wireless Communications, 2004.

[2] J. Liu, J. Reich, and F. Zhao, "Collaborative in-network processing for target tracking," EURASIP Journal on Applied Signal Processing, vol. 2003, pp. 378-391, March 2003.

[3] Y.-B. Ko and N. H. Vaidya, "Location-aided routing (LAR) in mobile ad hoc networks," MobiCom, 1998.

[4] M. Mauve, J. Widmer, and H. Hartenstein, "A survey on position-based routing in mobile ad- hoc networks," IEEE Network Magazine, 2001.

[5] T. Yan, T. He, and J. A. Stankovic, "Differentiated surveillance service for sensor networks," In Proceeding of First ACM Conference on Embedded Networked Sensor Systems (SenSys), 2003.

[6] B. H. Wellenhoff, H. Lichtenegger, and J. Collins, "Global positions system: Theory and practice," Fourth Edition, Springer Verlag, 1997.

[7] J. Hightower, G. Boriello, and R. Want, "Spoton: An indoor 3d location sensing technology based on RF signal strength," University of Washington CSE Report \#2000-02-02, 2000.

[8] P. Bahl and V. N. Padmanabhan, "Radar: An in-building RF-based user location and tracking system," IEEE InfoCom, 2000.

[9] N. Patwari and A. O. H. III, "Using proximity and quantized RSS for sensor localization in wireless networks," Workshop on Wireless Sensor Networks and Applications, 2003.

[10] N. B. Priyantha, A. Chakraborty, and H. Balakrishnan, "The cricket location-support system," In Proceedings of MOBICOM, 2000.

[11] K. Yedavalli, B. Krishnamachari, S. Ravula, and B. Srinivasan, "Ecolocation: A sequence based technique for RF-only localization in wireless sensor networks," The Fourth International Conference on Information Processing in Sensor Networks, April 2005.

[12] T. He, C. Huang, B. M. Blum, J. A. Stankovic, and T. Abdelzaher, "Range-free localization schemes for large scale sensor networks," MobiCom, 2003.

[13] T. He, C. Huang, B. M. Blum, J. A. Stankovic, and T. F. Abdelzaher, "Range-free localization and its impact on large scale sensor networks," ACM Transactions on Embedded Computing System (TECS), 2006.

[14] L. Hu and D. Evans, "Localization for mobile sensor networks," MobiCom, 2004.

[15] N. Priyantha, H. Balakrishnan, E. Demaine, and S. Teller, "Mobileassisted localization in wireless sensor networks," INFOCOM, 2005.

[16] R. Stoleru, T. He, and J. A. Stankovic, "Walking GPS: A practical solution for localization in manually deployed wireless sensor networks," 1st IEEE Workshop on Embedded Networked Sensors EmNetS-I., 2004.

[17] P. Pathirana, N. Bulusu, A. Savkin, and S. Jha, "Node localization using mobile robots in delay-tolerant sensor networks," IEEE Transactions on Mobile Computing, 2005.

[18] H. Lim and J. Hou, "Localization for anisotropic sensor networks," in Proc. of the 24th IEEE INFOCOM, 2005.

[19] P. Bergamo and G. Mazzini, "Localization in sensor networks with fading and mobility," IEEE PIMRC, 2002.

[20] T. S. Rappaport, "Wireless communications," Principles Practice, 1999.

[21] A. Galstyan, B. Krishnamachari, and S. P. K. Lerman, "Distributed online localization in sensor networks using a moving target," IPSN, 2004. 